\title{
The prognostic significance of vasohibin-1 expression in patients with prostate cancer
}

\author{
T Kosaka ${ }^{1,4}$, Y Miyazaki ${ }^{1,4}$, A Miyajima ${ }^{1}$, S Mikami ${ }^{2}$, Y Hayashi ${ }^{2}$, N Tanaka ${ }^{1}$, H Nagata ${ }^{1}$, E Kikuchi ${ }^{1}$, \\ K Nakagawa ${ }^{1}$, Y Okada $^{2}$, Y Sato ${ }^{3}$ and M Oya ${ }^{1}$ \\ ${ }^{1}$ Department of Urology, Keio University School of Medicine, 35 Shinanomachi, Shinjuku-ku, Tokyo 160-8582, Japan; \\ ${ }^{2}$ Department of Pathology, Keio University School of Medicine, 35 Shinanomachi, Shinjuku-ku, Tokyo 160-8582, Japan and \\ ${ }^{3}$ Department of Vascular Biology, Institute of Development, Aging, and Cancer, Tohoku University, 4-1, Seiryo-machi, Aoba-ku, \\ Sendai 980-9575, Japan
}

Background: We recently isolated vasohibin-1 (VASH1), a novel angiogenic molecule that is specifically expressed in activated vascular endothelial cells (ECs), and the status of VASH1 expression has been documented in various cancer angiogenesis. The aim of this study was to assess the prognostic value of VASH1 expression in prostate cancer (PCa).

Methods: In this study, we retrospectively analysed the clinical records and evaluated the VASH1 expression of tumour microvessels in 167 patients with PCa who underwent radical prostatectomy. We immunohistochemically examined the microvessels positive for anti-CD34 as microvessel density (MVD) and the microvessels with activated ECs positive for VASH1 density.

Results: We found that the VASH1 expression was restricted to ECs in the tumour stroma. VASH1 density was significantly associated with pathological T stage, Gleason score and MVD. The 5-year PSA recurrence-free survival rate was $58.8 \%$ in patients with higher VASH1 density $\left(\geqq 12\right.$ per $\left.\mathrm{mm}^{2}\right)$ and $89.1 \%$ in patients with lower VASH1 density $\left(<12\right.$ per $\left.\mathrm{mm}^{2}\right)$, respectively $(P<0.001)$. Microvessel density was not an independent predictor of PSA recurrence. Multivariate analysis revealed that high VASH1 density was an independent prognostic indicator of PSA recurrence $(P=0.007, \mathrm{HR}=2.950)$.

Conclusion: VASH1 density represents a clinically relevant predictor of patient prognosis and can be a new biomarker that would provide additional prognostic information in PCa.

Prostate cancer $(\mathrm{PCa})$ is one of the most commonly diagnosed malignant tumours in men and the second leading cause of cancerrelated deaths in the United States (Jemal et al, 2010). Nowadays, there are still few effective therapeutic options for advanced PCa (Scher and Sawyers, 2005; Chen et al, 2008). One of the most troublesome aspects of $\mathrm{PCa}$ is that androgen-dependent $\mathrm{PCa}$ inevitably progresses to highly aggressive and life-threatening castration-resistant prostate cancer after androgen ablation therapy. The key molecular events associated with PCa progression remains to be elucidated.

Angiogenesis, which is the formation of new blood vessel networks, not only plays a role in human normal development but also in pathophysiological conditions such as inflammation and cancer (Folkman, 1971; Sato, 2003). The development and establishment of blood supply has an important role in the development and growth of cancer cells by providing oxygen, nutrients and growth factors. In addition to its traditional role, angiogenesis is one of the potential pathways that contribute to solid tumour progression and metastasis by allowing cancer cells direct access to vessels. In general, angiogenesis is regulated by a balance between stimulatory and inhibitory factors (Sato and Sonoda, 2007, Sato, 2012). Molecules such as CD34, von Willebrand factor and vascular endothelial-cadherin, which are specifically expressed in vascular endothelial cells (ECs), could serve as biomarkers of angiogenesis (Weidner et al, 1991). One of the parameters of angiogenesis in neoplasms is microvessel density

\footnotetext{
*Correspondence: Dr T Kosaka; E-mail: takemduro@gmail.com
}

${ }^{4}$ These authors contributed equally to this work.

Received 7 February 2013; revised 21 March 2013; accepted 28 March 2013; published online 16 April 2013

(c) 2013 Cancer Research UK. All rights reserved 0007-0920/13 
(MVD). In PCa, several studies indicated that MVD served as a predictor of poorly differentiated tumours and biochemical failure after treatment (Weidner et al, 1993; Bettencourt et al, 1998; Borre et al, 1998; Rubin et al, 1999; Krupski et al, 2000; Josefsson et al, 2005; Concato et al, 2007, 2009; Kosaka et al, 2007). However, to date the prognostic role of MVD in PCa has not been fully characterised. This is because those angiogenic molecules are expressed in quiescent ECs as well as in activated ECs, and thus cannot reflect 'angiogenic activity' alone.

We recently isolated vasohibin-1 (VASH1), a novel angiogenic molecule, that is specifically expressed in ECs and upregulated by vascular endothelial growth factor (VEGF) and fibroblast growth factor 2 (FGF-2) (Watanabe et al, 2004; Kimura et al, 2009). Previous studies found that the expression of VASH1 was restricted to ECs of blood vessels in the tumour stroma and correlated with the expression of VEGF and FGF-2 in tumour cells (Hosaka et al, 2009). No one has ever characterised the expression of VASH1 in relation to tumour angiogenesis in PCa. In this study, we will evaluate whether the expression of VASH1 can serve as a more accurate biomarker of tumour angiogenesis compared with MVD.

We will examine the expression of VASH1 and MVD in PCa specimens acquired by primary surgery and investigate whether VASH1 expression reflects angiogenic activity in $\mathrm{PCa}$ and if this is related to the clinical outcome.

\section{MATERIALS AND METHODS}

Patients selection. Samples of archival paraffin-embedded tissue sections and clinicopathological features were obtained from 167 patients with PCa diagnosed and operated on at Keio University hospital. One hundred and sixty-seven patients underwent curative surgery that included radical prostatectomy for localised PCa between January 2000 and December 2003. None of the patients had received hormonal treatment before the operation. The characteristics of these patients are shown in Table 1. After radical prostatectomy, patients were followed by serum PSA level and imaging studies. Prostate-specific antigen relapse was defined by an elevation of serum PSA level at three consecutive measurements. Histology of the specimens was evaluated by two independent pathologists using haematoxylin- and eosin-stained tissue preparations.

Tissue samples and immunohistochemistry. All the tissue samples were fixed in $10 \%$ formalin, embedded in paraffin and cut into $4-\mu$ m-thick sections. All pathological specimens were reviewed again by genitourinary pathologists to unify the reproducibility of the diagnosis. As for the pathologic stage, all neoplasms were classified according to the 2006 TNM staging system. All study participants provided their informed consent, and the study design was approved by the ethics review board of Keio University. We carried out immunohistochemical staining for VASH1 and CD34 (as markers of vascular ECs). Tissue sections were deparaffinised in xylene, and hydrated by immersion in graded alcohols and finally in distilled water. After antigen retrieval was performed, endogenous peroxidase activity was blocked by incubation in $0.3 \%$ hydrogen peroxidase for $20 \mathrm{~min}$ at room temperature. The tissue sections were then incubated with a blocking solution of $5 \%$ dry milk in PBS. They were stained for $60 \mathrm{~min}$ at room temperature with primary antibodies, followed by staining for $30 \mathrm{~min}$ at room temperature with secondary antibodies. The primary antibodies were all mouse monoclonal antibodies (mAbs): anti-human VASH1 mAb diluted at 1:400 and anti-CD34 (Nichirei Biosciences, Tokyo, Japan) diluted at 1:200. We previously described a mouse $\mathrm{mAb}$ against a synthetic peptide corresponding to the 286-299 amino-acid sequence of VASH1 (Watanabe et al, 2004). The specificity of this antibody, which detects VASH1, has been documented in our previous studies (Watanabe et al, 2004; Yoshinaga et al, 2008, 2011; Hosaka et al, 2009; Kimura et al, 2009; Tamaki et al, 2009, 2010; Miyashita et al, 2012; Miyazaki et al, 2012). After washing with PBS, the tissue sections were incubated with secondary antibodies (Histofine Simple Stain MAX PO (M); Nichirei Biosciences). Colour was developed with $3,3^{\prime}$-diaminobenzamine tetrahydrochloride in $50 \mathrm{~mm}$ Tris- $\mathrm{HCl}$ ( $\mathrm{pH} 7.5$ ) containing $0.005 \%$ hydrogen peroxide. The sections were counterstained with haematoxylin. The positive control slide CD34 antigen was prepared from paraffin-fixed bladder cancer tissue with high MVD. The appropriate negative controls slides for CD34 antigen and VASH1 were prepared by substituting the primary antibody with the immune globulin fraction of nonimmune mouse serum at the same concentration in each staining run.

\section{Table 1. Correlation of clinicopathological parameters and MVD or VASH1 expression in the 167 study patients}

\begin{tabular}{|c|c|c|c|c|c|}
\hline Characteristic & No. of patients (\%) & MVD (mean \pm s.d.) & $P$-value & $\begin{array}{l}\text { VASH1 density } \\
\text { (mean } \pm \text { s.d.) }\end{array}$ & $\boldsymbol{P}$-value \\
\hline \multicolumn{6}{|l|}{ Age (years) } \\
\hline $\begin{array}{l}<65 \\
\geqq 65\end{array}$ & $\begin{array}{l}99(59.3) \\
68(40.7)\end{array}$ & $\begin{array}{l}133.1 \pm 58.3 \\
115.6 \pm 41.2\end{array}$ & 0.201 & $\begin{array}{c}9.3 \pm 7.4 \\
10.8 \pm 7.2\end{array}$ & 0.101 \\
\hline \multicolumn{6}{|l|}{ PSA } \\
\hline $\begin{array}{l}<15 \\
\geqq 15\end{array}$ & $\begin{array}{l}138(82.6) \\
29(17.4)\end{array}$ & $\begin{array}{l}120.5 \pm 47.5 \\
133.7 \pm 67.9\end{array}$ & 0.588 & $\begin{array}{c}9.4 \pm 6.8 \\
12.3 \pm 8.9\end{array}$ & 0.139 \\
\hline \multicolumn{6}{|l|}{ Gleason score } \\
\hline $\begin{array}{l}\leqq 6 \\
\geqq 7\end{array}$ & $\begin{array}{l}69(41.3) \\
98(58.7)\end{array}$ & $\begin{array}{l}105.8 \pm 42.2 \\
134.6 \pm 54.4\end{array}$ & $<0.001$ & $\begin{array}{c}7.5 \pm 5.6 \\
11.6 \pm 7.9\end{array}$ & $<0.001$ \\
\hline \multicolumn{6}{|c|}{ Pathological Tstage } \\
\hline $\begin{array}{l}\leqq \text { pT2 } \\
\geqq \text { pT3 }\end{array}$ & $\begin{array}{c}113(67.7) \\
54(32.3)\end{array}$ & $\begin{array}{l}111.2 \pm 46.3 \\
146.8 \pm 54.3\end{array}$ & $<0.001$ & $\begin{array}{c}8.6 \pm 6.5 \\
12.6 \pm 8.2\end{array}$ & $<0.001$ \\
\hline
\end{tabular}


CD34
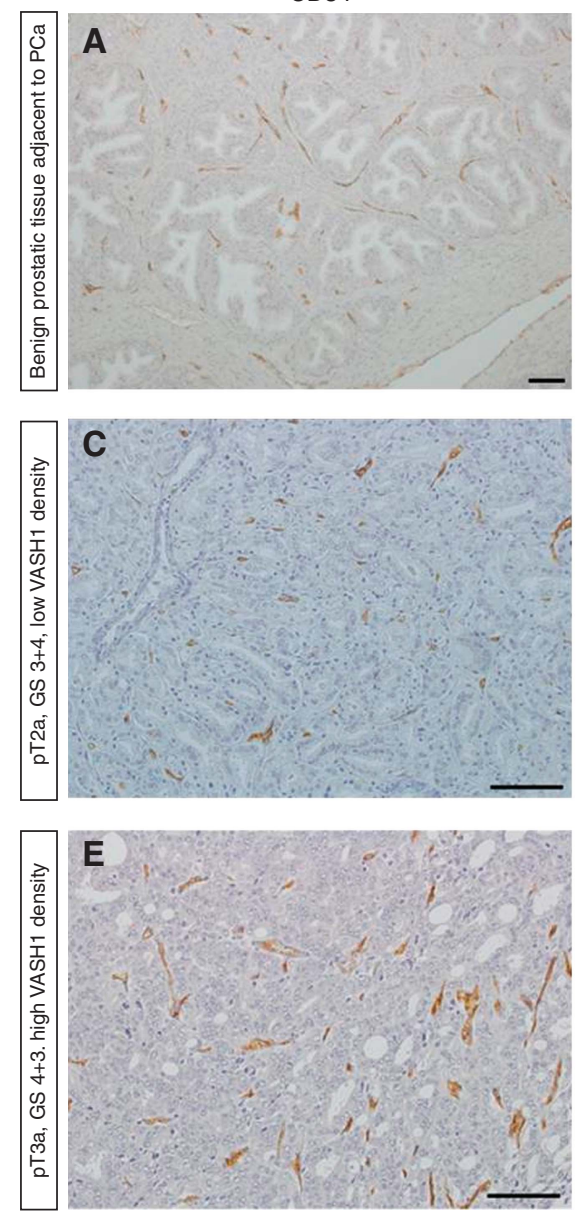

Vasohibin-1
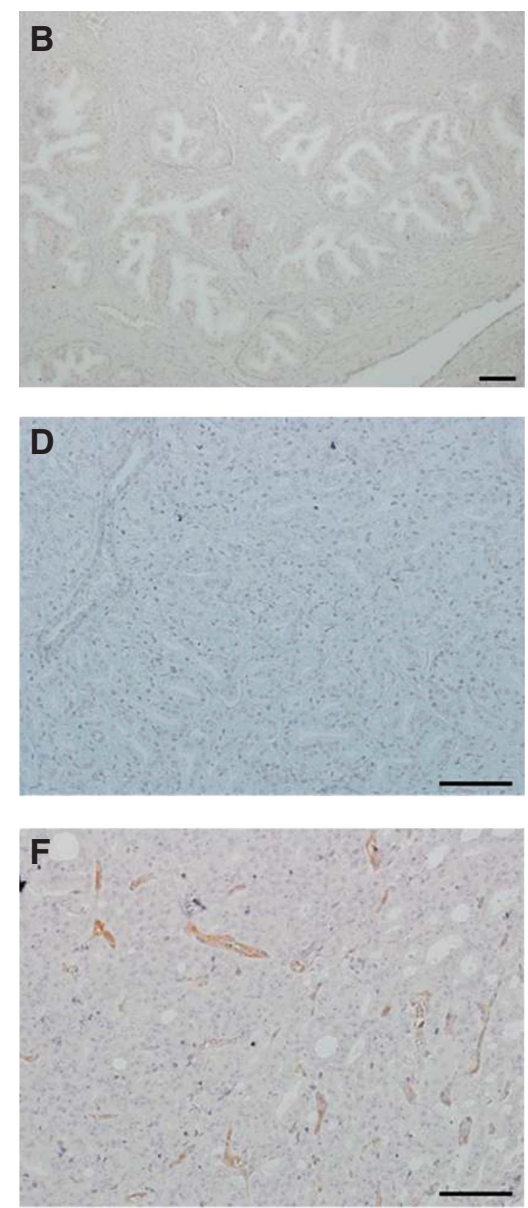

Figure 1. Immunostaining for CD34 (A, C and E) and VASH1 (B, D and F) in PCa. CD34 staining (A) and VASH1 staining (B) of vascular ECs in benign prostatic tissue adjacent to cancerous tissue. VASH1 staining of vascular ECs was negative or negligible. Low $\mathrm{pT}$ stage and PCa with low VASH1 density ( $\mathbf{C}$ and $\mathbf{D}$ ) or high pT stage with high VASH1 density ( $\mathbf{E}$ and $\mathbf{F})$. Bar $=0.1 \mathrm{~mm}$.

Evaluation of immunostaining. Two authors independently evaluated immunoreactivity. They were blinded to the clinical course of the patients and the average of the numbers counted by the two investigators was used for subsequent analyses. Olympus IX71 (Olympus, Tokyo, Japan) was used for the analysis. The microvessels within the tumour were counted. Microvessels were identified based on their architecture, lumen lined by ECs and complemented by positivity of the ECs for anti-CD34 after scanning the immunostained section at low magnification $(\times 40$ and $\times 100)$. The areas with the highest number of distinctly highlighted microvessels were selected, and these were counted at high magnification $(\times 200)$. We evaluated at least six areas in high-power fields and selected one area with the highest number of vessels. Any immunostained EC or cluster separated from adjacent vessels was counted as a single microvessel, even in the absence of vessel lumen. Each single count was defined as the highest number of microvessels identified at the 'hot spot' as shown previously (Mikami et al, 2006; Kosaka et al, 2007; Shirotake et al, 2011; Yoshinaga et al, 2011). The highest number of microvessels in the hot spot was counted for MVD. Vasohibin-1-positive signals were counted in the 'hot spot' in which the highest number of vessels positive for anti-CD34 was identified. We regarded the number of VASH1-positive signals per $\mathrm{mm}^{2}$ as 'VASH1 density' (Tamaki et al, 2009, 2010; Yoshinaga et al, 2011). The median values of MVD and VASH1 density were 119.5 and 9.9 per $\mathrm{mm}^{2}$, respectively. We used a median MVD of $\geqq 120$ per $\mathrm{mm}^{2}$ and a VASH1 density of $\geqq 12$ per $\mathrm{mm}^{2}$ as the cutoff levels.
Statistical analysis. The associations between each clinicopathological parameter and VASH1 density of the tumour were analysed. These associations were validated using $\chi^{2}$ test or Mann-Whitney $U$-test. Biochemical recurrence-free survival was estimated using the Kaplan-Meier method and was compared by using the log-rank test. Multivariate analysis was performed using the Cox proportional hazard model. Differences among groups were regarded as significant when $P<0.05$. These analyses were performed with the SPSS version 18.0 statistical software package (IBM corporation, New York, NY, USA).

\section{RESULTS}

Patient characteristics and VASH1 expression in PCa. Table 1 shows the clinicopathological characteristics of the patients and their association with MVD or VASH1 density in our study population. The median age of the patients was 66.6 years (range 46-75 years). Pathological T (pT) stage was $\leqq \mathrm{pT} 2$ in 113 cases $(67.7 \%)$ and $\geqq \mathrm{pT} 3$ in 54 cases $(32.3 \%)$. Gleason score (GS) was $\leqq$ 6 in 69 cases $(41.3 \%)$ and $\geqq 7$ in 98 cases $(58.7 \%)$. During a median follow-up of 4.9 years, 48 patients (28.7\%) experienced PSA recurrence. To elucidate the biological significance of VASH1 in $\mathrm{PCa}$, we examined the expression of VASH1 by immunohistochemical staining (Figure 1). Although CD34 staining of vascular ECs in benign prostatic tissue adjacent to cancerous tissue was 


\begin{tabular}{|c|c|c|c|}
\hline & \multicolumn{3}{|c|}{ Recurrence-free survival } \\
\hline & Univariate & Multivariat & \\
\hline Characteristic & $\boldsymbol{P}$-value & $\mathrm{HR}(95 \% \mathrm{Cl})$ & $\boldsymbol{P}$-value \\
\hline $\begin{array}{l}\text { Age (years) } \\
<65 \\
\geqq 65\end{array}$ & 0.525 & & \\
\hline PSA & $<0.001$ & & \\
\hline $\begin{array}{l}<15 \\
\geqq 15\end{array}$ & & & \\
\hline Gleason score & 0.073 & & \\
\hline $\begin{array}{l}\leqq 6 \\
\geqq 7\end{array}$ & & & \\
\hline Pathological T stage & $<0.001$ & & $<0.001$ \\
\hline $\begin{array}{l}\leqq \text { pT2 } \\
\geqq \text { pT3 }\end{array}$ & & $4.667(2.358-9.234)$ & \\
\hline MVD & $<0.001$ & & \\
\hline $\begin{array}{l}<120 \text { per } \mathrm{mm}^{2} \\
\geqq 120 \text { per } \mathrm{mm}^{2}\end{array}$ & & & \\
\hline VASH1 density & $<0.001$ & & 0.007 \\
\hline $\begin{array}{l}<12 \text { per } \mathrm{mm}^{2} \\
\geqq 12 \text { per } \mathrm{mm}^{2}\end{array}$ & & $2.950(1.349-6.449)$ & \\
\hline
\end{tabular}

positive as shown previously (Figure 1A), VASH1 staining of vascular ECs was negative or negligible (Figure 1B). Vasohibin-1 staining of vascular ECs was negative or negligible in low pT stage and low GS PCa (Figure 1D). On the other hand, in high pT stage PCa, strong VASH1 staining of vascular ECs was detected in many cases (Figure 1F). Strong VASH1 staining was observed in ECs of microvessels in the tumour lesion of high pT stage and high GS specimens. VASH1 staining of vascular ECs was negative or negligible in large-size vessels of the tumour detected by CD34.

The average MVD and VASH1 density (counts per $\mathrm{mm}^{2}$ ) were $123 \pm 51.6$ and $9.9 \pm 7.3$ in 167 patients, respectively (Table 1). Patients with high GS tumours $(P<0.001)$ or $\geqq$ pT3 $(P<0.001)$ had significantly higher levels of both MVD and VASH1 density. As it has been reported that VASH1 associates with CD34, we also investigated the relationship between VASH1 and CD34 expression. Using Spearman's correlation coefficient test, we detected a significant positive correlation between MVD and VASH1 density in microvessels in the tumour $(\rho=0.504$, $P<0.001)$.

Prognostic significance of VASH1 expression in PCa patients. We performed univariate and multivariate analysis to determine the indicators for subsequent PSA recurrence following surgery (Table 2). Univariate analysis revealed that high PSA concentration $(P<0.001)$, high pT stage $(P<0.001)$, high MVD $(P<0.001)$ and high VASH1 density $\left(\geqq 12\right.$ per $\left.\mathrm{mm}^{2}\right)(P<0.001)$ were significant predictors of tumour recurrence. Multivariate analysis showed that high pT stage $(P<0.001, \mathrm{HR}=4.667)$ and high VASH1 density $(P=0.007, \mathrm{HR}=2.950)$ were also independent predictors of PSA recurrence. A high level of MVD was not an independent predictor of PSA recurrence. Table 3 shows the association between the level of VASH1 density and clinic-pathological characteristics in 167 patients. High VASH1 density was significantly associated with GS
Table 3. Clinicopathological parameters in 167 patients according to the level of the VASH1 density

No. of patients (\%)

\begin{tabular}{|c|c|c|c|}
\hline Characteristic & $\begin{array}{l}\text { Patients with } \\
\text { VASH1 density } \\
<12 \text { per } \mathrm{mm}^{2}\end{array}$ & $\begin{array}{l}\text { Patients with } \\
\text { VASH1 density } \\
\geqq 12 \text { per } \mathrm{mm}^{2}\end{array}$ & $\boldsymbol{P}$-value \\
\hline No. of patients & 83 & 84 & \\
\hline \multicolumn{4}{|l|}{ Age (years) } \\
\hline $\begin{array}{l}<65 \\
\geqq 65\end{array}$ & $\begin{array}{l}53(63.9) \\
30(36.1)\end{array}$ & $\begin{array}{l}46(54.8) \\
38(45.2)\end{array}$ & 0.271 \\
\hline \multicolumn{4}{|l|}{ PSA } \\
\hline $\begin{array}{l}<15 \\
\geqq 15\end{array}$ & $\begin{array}{l}72(86.7) \\
11(13.3)\end{array}$ & $\begin{array}{l}66(78.6) \\
18(21.4)\end{array}$ & 0.220 \\
\hline \multicolumn{4}{|l|}{ Gleason score } \\
\hline $\begin{array}{l}\leqq 6 \\
\geqq 7\end{array}$ & $\begin{array}{l}48(57.8) \\
35(42.2)\end{array}$ & $\begin{array}{l}21(25.0) \\
63(75.0)\end{array}$ & $<0.001$ \\
\hline \multicolumn{4}{|c|}{ Pathological T stage } \\
\hline $\begin{array}{l}\leqq \text { pT2 } \\
\geqq \text { pT3 }\end{array}$ & $\begin{array}{l}65(78.3) \\
18(21.7)\end{array}$ & $\begin{array}{l}48(57.1) \\
36(42.9)\end{array}$ & 0.005 \\
\hline \multicolumn{4}{|l|}{ MVD } \\
\hline $\begin{array}{l}<120 \text { per } \mathrm{mm}^{2} \\
\geqq 120 \text { per } \mathrm{mm}^{2}\end{array}$ & $\begin{array}{l}62(74.7) \\
21(25.3) \\
\end{array}$ & $\begin{array}{l}21(25.0) \\
63(75.0) \\
\end{array}$ & $<0.001$ \\
\hline $\begin{array}{l}\text { Abbreviations: MVD } \\
\text { vasohibin-1. }\end{array}$ & microvessel density; PS & $=$ prostate-specific antige & ; $\mathrm{VASH} 1=$ \\
\hline
\end{tabular}

$(P<0.001)$, pT stage $(P=0.005)$ and MVD $(P<0.001)$. The 5-year Kaplan-Meier PSA recurrence-free survival rate was $58.8 \%$ in patients with high VASH1 density compared with $89.1 \%$ $(P<0.001)$ in their counterparts (Figure 2A).

Risk stratification for PCa according to pT stage and VASH1 density. We distributed the patients into three different groups according to pT stage and VASH1 density, which were the two statistically significant variables found by the multivariate Cox regression analysis (Figure $2 \mathrm{C}$ ). The relative risk of death was calculated with the formula, exp $(1.540 \times \mathrm{pT}$ stage $+1.082 \times$ VASH1 density) for PSA recurrence-free survival. In this equation, the pT stage equaled 1 if the pT stage was pT3 or more, and it equaled 0 if the pT stage was pT2 or less. VASH1 density equaled 1 if VASH1 density was $\geqq 12$ per $\mathrm{mm}^{2}$ and 0 if $<12$ per $\mathrm{mm}^{2}$. On the basis of the relative risk of death, patients with PCa were divided into three risk groups: low (relative risk of PSA recurrence $=1)$, intermediate $(8.02-12.7$ for PSA recurrence-free survival) and high (13.8 for PSA recurrence-free survival). According to the risk stratification for PCa based on prognostic factors, 65 patients $(38.9 \%)$ were in the low-risk group (low pT stage and low VASH1 density), 66 patients (39.5\%) were in the high-risk group (high pT stage and high VASH1 density) and 36 patients $(21.6 \%)$ were in the intermediate-risk group (all others). The 5-year PSA recurrence-free survival was $98.1 \%$ in the low-risk group, $69.7 \%$ in the intermediate-risk group and $33.7 \%$ in the highrisk group, respectively. The differences among the groups were significant $(P<0.001$ in PSA recurrence-free survival and for low$v s$ intermediate-risk group, $P<0.001$ for low- $v s$ high-risk group 

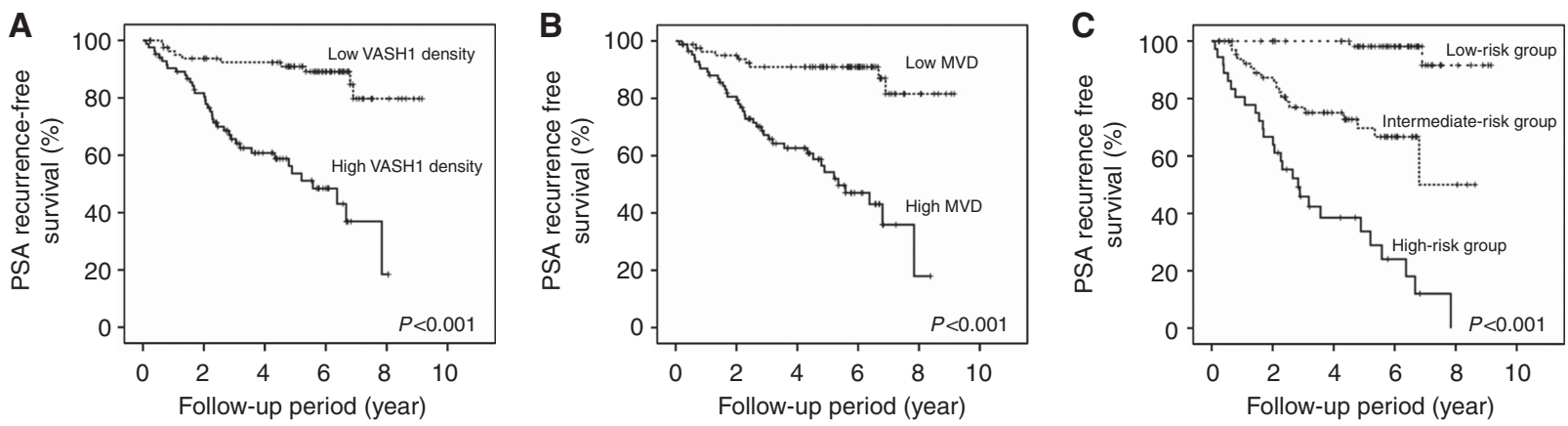

Figure 2. Kaplan-Meier curves of PSA recurrence-free survival of the patients after surgery for PCa according to VASH1 density (A) or MVD (B). Kaplan-Meier curves of PSA recurrence-free survival $(\mathbf{C})$ of the patients after surgery for PCa according to PT stage and VASH1 density stratified according to three risk groups. Low-risk group consisted of patients with $\leq$ pT2 and low VASH1 density. High-risk group consisted of those with $\geq$ PT3 and high VASH1 density. All others were included in the intermediate-risk group.
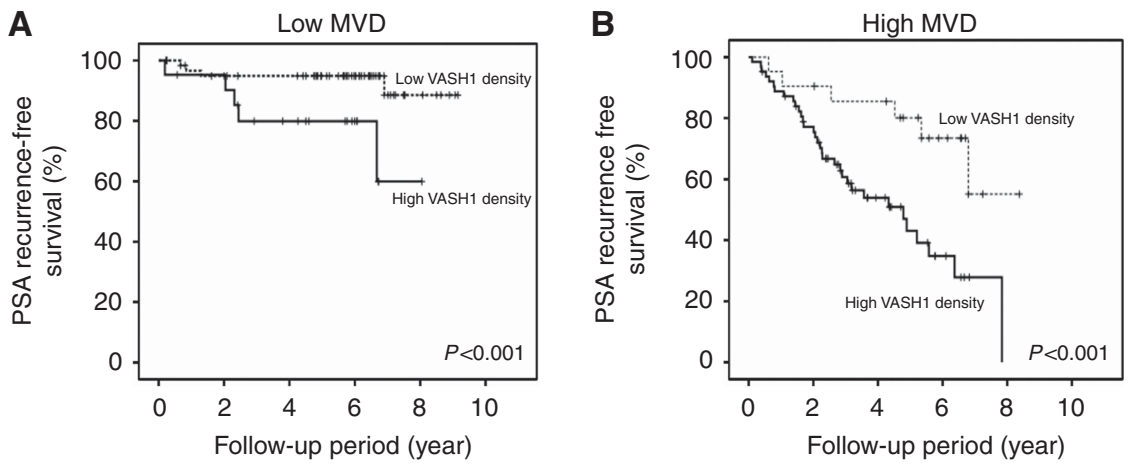

Figure 3. Kaplan-Meier curves of PSA recurrence-free survival of the patients among low (A) or high (B) MVD groups according to VASH1 density. The patients with high MVD and high VASH1 density had a significantly poorer prognosis than the counterpart group $(P<0.001)$.

and $P<0.001$ for intermediate- $v s$ high-risk group in PSA recurrence-free survival).

\section{Microvessel density could be divided into two subclasses for PSA} recurrence according to VASH1 density in PCa patients. Univariate analysis revealed that high MVD was one of the significant predictors of PSA recurrence (Figure 2B), while multivariate analysis showed that high MVD was not an independent predictor of PSA recurrence. Then, we re-evaluated univariate analysis of MVD according to high or low VASH1 density (Figure 3). In the low MVD group, 21 patients (25.3\%) had high VASH1 density and 62 patients (74.7\%) had low VASH1 density (Figure 3A). The 5-year PSA recurrence-free survival rate in the low MVD group was $79.9 \%$ for those with high VASH1 density and $94.9 \%$ for those with low VASH1 density. The difference between the groups was statistically significant $(P<0.001)$. In the high MVD group, 63 patients $(75.0 \%)$ had high VASH1 density and 21 patients (25.0\%) had low VASH1 density (Figure 3B). The 5-year PSA recurrence-free survival rate in the high MVD group was $43.1 \%$ for those with high VASH1 density and $80.1 \%$ for those with low VASH1 density. The differences were significant $(P<0.001)$. These results indicated that MVD in PCa could be subdivided into two subclasses for PSA recurrence according to VASH1 density.

\section{DISCUSSION}

In this study, we retrospectively evaluated the impact of VASH1 expression by immunohistochemical staining in a series of patients with PCa treated in a single centre. Our results suggested that VASH1 expression was a prognostic indicator in addition to other standard factors such as pT stage. High VASH1 density was related to shorter patient PSA recurrence-free survival. To the best of our knowledge, this is the first study evaluating the prognostic value of VASH1 expression in patients with prostate cancer.

Angiogenesis has a critical role in tumour growth and metastasis (Folkman, 1971; Chung et al, 2010). Recent studies revealed significant roles for angiogenesis in the prediction of survival in patients with different malignancies (Sato, 2003, 2012; Chung et al, 2010). One of the biomarkers that could reflect angiogenic aggressiveness was MVD (Weidner et al, 1991; Bochner et al, 1995; Kosaka et al, 2007; Shirotake et al, 2011). Several studies on PCa indicated that the status of MVD was associated with clinical features, such as GS and pathological stage, and could be an independent prognostic factor of patient survival (Weidner et al, 1993; Bettencourt et al, 1998; Borre et al, 1998; Rubin et al, 1999; Krupski et al, 2000; Josefsson et al, 2005; Concato et al, 2007, 2009; Kosaka et al, 2007). However, to date evidence of the prognostic role of MVD in $\mathrm{PCa}$ is contradictory, suggesting that the prognostic impact of MVD might be controversial. In this study, univariate analysis reveled that there was significant association between MVD and PSA recurrence. However, multivariate analysis including VASH1 density showed that MVD did not have a prognostic significance in PCa progression. One of the reasons might be because MVD corresponds to the number of accomplished vessels and includes vessels without the potential of neovascularisation in $\mathrm{PCa}$. Our previous reports showed that VASH1 has been isolated from VEGF-inducible genes in ECs present in newly formed blood vessels behind the sprouting front where angiogenesis terminates (Watanabe et al, 2004; Sato and Sonoda, 2007; Hosaka et al, 2009). Recently, we have reported that histologic evidence of VASH1 expression has been found in 
samples from patients with endometrial carcinoma (Yoshinaga et al, 2008), breast cancer (Tamaki et al, 2009, 2010) and cervical carcinoma (Yoshinaga et al, 2011). Our reports indicated that VASH1 expression was associated with tumour grade and histological type of carcinomas. Moreover, it was reported that VASH1 expression tended to be concordant with MVD, although partial dissociation was observed in some patients with breast carcinoma. VASH1 expression was significantly higher in invasive breast carcinoma, although no significant difference was observed in the level of MVD between patients with invasive disease or not (Tamaki et al, 2010). These studies suggested that an evaluation of the number of VASH1-positive vessels may become one of the prognostic biomarkers for metastasis and prognosis. These results indicate that VASH1 could become a new molecular biomarker of the angiogenic heterogeneity of tumours. In this study, we demonstrated that the prognostic value of MVD depends on the level of VASH1 density.

Our study demonstrated that PCa with a higher number of VASH1-positive vessels tended to have a poor prognosis. We found a significant correlation among VASH1 density, GS and pT stage. Multivariate analysis showed that high VASH1 density was an independent prognostic factor, suggesting that the status of VASH1 density could serve as a biomarker of the malignant potential of tumour angiogenesis. These results suggest that the level of VASH1 expression may influence the clinical course of prostate cancer progression. Moreover, VASH1 may become a molecular target in PCa patients.

Using VASH1 density and other independent indicators, we established a prognostic risk stratification for PCa. Patients were stratified into three groups according to statistical modelling based on the relative risk associated with the prognostic indicators derived from multivariate analysis. As shown in Figure 2C among patients with $\mathrm{PCa}$, the prognosis of patients with higher $\mathrm{pT}$ stage and high VASH1 density was worse than that of the other groups $(P<0.001)$. This stratification made it possible to predict PSA recurrence more accurately, suggesting more appropriate follow-up including PSA follow-up interval.

In conclusion, these results indicated that VASH1 can serve as a new biomarker for predicting $\mathrm{PCa}$ progression and could become a molecular target in $\mathrm{PCa}$, especially for targeting tumour angiogenesis.

\section{ACKNOWLEDGEMENTS}

We thank colleagues from the Department of Vascular Biology, Institute of Development, Aging and Cancer, Tohoku University for their excellent technical assistance in purifying anti-human VASH1 mAb. This work was supported in part by grants-in-aid for Scientific Research from the Ministry of Education, Culture, Sports, Science, and Technology of Japan, by the Prostate Research Fund in Japan, the Japan Urological Association (5th Young Research Grant 2011 to TK) and the Cooperative Research Project Program 2011, 2012 of Joint Usage/Research Center at the Institute of Development, Aging and Cancer, Tohoku University.

\section{CONFLICT OF INTEREST}

The authors declare no conflict of interest.

\section{REFERENCES}

Bettencourt MC, Bauer JJ, Sesterhenn IA, Connelly RR, Moul JW (1998) $\mathrm{CD} 34$ immunohistochemical assessment of angiogenesis as a prognostic marker for prostate cancer recurrence after radical prostatectomy. J Urol 160: 459-465.

Bochner BH, Cote RJ, Weidner N, Groshen S, Chen SC, Skinner DG, Nichols PW (1995) Angiogenesis in bladder cancer: relationship between microvessel density and tumor prognosis. J Natl Cancer Inst 87: $1603-1612$.

Borre M, Offersen BV, Nerstrom B, Overgaard J (1998) Microvessel density predicts survival in prostate cancer patients subjected to watchful waiting. Br J Cancer 78: 940-944.

Chen Y, Sawyers CL, Scher HI (2008) Targeting the androgen receptor pathway in prostate cancer. Curr Opin Pharmacol 8: 440-448.

Chung AS, Lee J, Ferrara N (2010) Targeting the tumour vasculature: insights from physiological angiogenesis. Nat Rev Cancer 10: 505-514.

Concato J, Jain D, Li WW, Risch HA, Uchio EM, Wells CK (2007) Molecular markers and mortality in prostate cancer. BJU Int 100: 1259-1263.

Concato J, Jain D, Uchio E, Risch H, Li WW, Wells CK (2009) Molecular markers and death from prostate cancer. Ann Intern Med 150: 595-603.

Folkman J (1971) Tumor angiogenesis: therapeutic implications. N Engl J Med 285: 1182-1186.

Hosaka T, Kimura H, Heishi T, Suzuki Y, Miyashita H, Ohta H, Sonoda H, Moriya T, Suzuki S, Kondo T, Sato Y (2009) Vasohibin-1 expression in endothelium of tumor blood vessels regulates angiogenesis. Am J Pathol 175: $430-439$.

Jemal A, Siegel R, Xu J, Ward E (2010) Cancer statistics, 2010. CA Cancer J Clin 60: 277-300.

Josefsson A, Wikstrom P, Granfors T, Egevad L, Karlberg L, Stattin P, Bergh A (2005) Tumor size, vascular density and proliferation as prognostic markers in GS 6 and GS 7 prostate tumors in patients with long follow-up and non-curative treatment. Eur Urol 48: 577-583.

Kimura H, Miyashita H, Suzuki Y, Kobayashi M, Watanabe K, Sonoda H, Ohta H, Fujiwara T, Shimosegawa T, Sato Y (2009) Distinctive localization and opposed roles of vasohibin-1 and vasohibin-2 in the regulation of angiogenesis. Blood 113: 4810-4818.

Kosaka T, Miyajima A, Takayama E, Kikuchi E, Nakashima J, Ohigashi T, Asano T, Sakamoto M, Okita H, Murai M, Hayakawa M (2007) Angiotensin II type 1 receptor antagonist as an angiogenic inhibitor in prostate cancer. Prostate 67: 41-49.

Krupski T, Petroni GR, Frierson Jr. HF, Theodorescu JU (2000) Microvessel density, p53, retinoblastoma, and chromogranin A immunohistochemistry as predictors of disease-specific survival following radical prostatectomy for carcinoma of the prostate. Urology 55: 743-749.

Mikami S, Oya M, Mizuno R, Murai M, Mukai M, Okada Y (2006) Expression of Ets-1 in human clear cell renal cell carcinomas: implications for angiogenesis. Cancer Sci 97: 875-882.

Miyashita H, Watanabe T, Hayashi H, Suzuki Y, Nakamura T, Ito S, Ono M, Hoshikawa Y, Okada Y, Kondo T, Sato Y (2012) Angiogenesis inhibitor vasohibin-1 enhances stress resistance of endothelial cells via induction of SOD2 and SIRT1. PLoS One 7: e46459.

Miyazaki Y, Kosaka T, Mikami S, Kikuchi E, Tanaka N, Maeda T, Ishida M, Miyajima A, Nakagawa K, Okada Y, Sato Y, Oya M (2012) The prognostic significance of vasohibin-1 expression in patients with upper urinary tract urothelial carcinoma. Clin Cancer Res 18: 4145-4153.

Rubin MA, Buyyounouski M, Bagiella E, Sharir S, Neugut A, Benson M, de la Taille A, Katz AE, Olsson CA, Ennis RD (1999) Microvessel density in prostate cancer: lack of correlation with tumor grade, pathologic stage, and clinical outcome. Urology 53: 542-547.

Sato Y (2003) Molecular diagnosis of tumor angiogenesis and anti-angiogenic cancer therapy. Int J Clin Oncol 8: 200-206.

Sato Y, Sonoda H (2007) The vasohibin family: a negative regulatory system of angiogenesis genetically programmed in endothelial cells. Arterioscler Thromb Vasc Biol 27: 37-41.

Sato Y (2012) The vasohibin family: Novel regulators of angiogenesis. Vascul Pharmacol 56: 262-266.

Scher HI, Sawyers CL (2005) Biology of progressive, castration-resistant prostate cancer: directed therapies targeting the androgen-receptor signaling axis. J Clin Oncol 23: 8253-8261.

Shirotake S, Miyajima A, Kosaka T, Tanaka N, Maeda T, Kikuchi E, Oya M (2011) Angiotensin II type 1 receptor expression and microvessel density in human bladder cancer. Urology 77: 1009, e19-e25.

Tamaki K, Moriya T, Sato Y, Ishida T, Maruo Y, Yoshinaga K, Ohuchi N, Sasano H (2009) Vasohibin-1 in human breast carcinoma: a potential negative feedback regulator of angiogenesis. Cancer Sci 100: 88-94. 
Tamaki K, Sasano H, Maruo Y, Takahashi Y, Miyashita M, Moriya T, Sato Y, Hirakawa H, Tamaki N, Watanabe M, Ishida T, Ohuchi N (2010) Vasohibin-1 as a potential predictor of aggressive behavior of ductal carcinoma in situ of the breast. Cancer Sci 101: 1051-1058.

Watanabe K, Hasegawa Y, Yamashita H, Shimizu K, Ding Y, Abe M, Ohta H, Imagawa K, Hojo K, Maki H, Sonoda H, Sato Y (2004) Vasohibin as an endothelium-derived negative feedback regulator of angiogenesis. J Clin Invest 114: 898-907.

Weidner N, Semple JP, Welch WR, Folkman J (1991) Tumor angiogenesis and metastasis - correlation in invasive breast carcinoma. $N$ Engl $J$ Med 324: 1-8.

Weidner N, Carroll PR, Flax J, Blumenfeld W, Folkman J (1993) Tumor angiogenesis correlates with metastasis in invasive prostate carcinoma. Am J Pathol 143: 401-409.
Yoshinaga K, Ito K, Moriya T, Nagase S, Takano T, Niikura H, Sasano H, Yaegashi N, Sato Y (2011) Roles of intrinsic angiogenesis inhibitor, vasohibin, in cervical carcinomas. Cancer Sci 102: 446-451. Yoshinaga K, Ito K, Moriya T, Nagase S, Takano T, Niikura H, Yaegashi N, Sato Y (2008) Expression of vasohibin as a novel endothelium-derived angiogenesis inhibitor in endometrial cancer Cancer Sci 99: 914-919.

This work is published under the standard license to publish agreement. After 12 months the work will become freely available and the license terms will switch to a Creative Commons AttributionNonCommercial-Share Alike 3.0 Unported License. 